\title{
Electrolytic colouring of anodized aluminium on tin basis
}

\author{
Barbora Šopová, Matilda Zemanová \\ Institute of Inorganic Chemistry, Technology and Materials, \\ Faculty of Chemical and Food Technology STU in Bratislava, \\ Radlinského 9, 81237 Bratislava, Slovak Republic \\ xsopova@is.stuba.sk
}

\begin{abstract}
The aim of the study was to find parameters of electrolytic colouring on tin basis to form uniform black coatings on anodized aluminium. A two steps electrolytic process consisting of aluminium anodization in a sulphuric acid electrolyte and colouring in tin acidic electrolyte was used. Among parameters influencing the colouring process, AC colouring voltage, composition of the counter electrode and agitation of the colouring electrolyte were studied. Spectrocolorimetry was applied to analyse the quality of the colouring. Thickness of the coloured and sealed anodized specimens was also evaluated. Optimal parameters for uniform colouring of anodized specimens were found. Unfortunately, colouring electrolyte on tin basis is susceptible to oxidation which negatively influences the colouring.
\end{abstract}

Keywords: aluminium; anodic oxidation; tin based electrolytic colouring; hydrothermal sealing; $L^{*} a^{*} b^{*}$ system

\section{Introduction}

Architecture, aircraft and nowadays automotive industry require surface treatment of metals applying conversion coatings which are characterised by corrosion resistance and adhesion improvement.

Aluminium and its alloys are used due to their excellent mechanical, chemical and electrical properties. Aluminium reacts with oxygen in the atmosphere and forms a natural aluminium oxide film on the surface with the thickness of about tenths of nm. The oxide layer can be enhanced several times by anodic oxidation (anodization). Sulphuric acid electrolyte is commonly used for the anodization (Wernick et al., 1987; Brace et al., 1979). The anodic oxide film consists of a thin barrier layer formed directly on the metal and a thick oxide layer with hexagonally shaped cells and a central pore as it can be seen in Fig. 1. Advantage of the sulphuric acid electrolyte consists in low power consumption and high reliability (Švorc, 2005; Michna, 2016). Electrolytic colouring uses mostly alternating current (AC) to deposit metal into the pores. Metals like tin, nickel, cobalt, etc., are used. Tin acidic colouring electrolytes are employed mostly in the automotive industry. The main reason for tin colouring is to meet toxicity requirements and to achieve good throwing power, minimal sensitivity to $\mathrm{pH}$ and bath contamination; it is also relatively easy to operate. The main disadvantage of the bath is the electrolyte oxidation in the atmosphere (Tsangaraki-Kaplanoglou et al., 2006)

An important part of the surface treatment of the coloured anodized aluminium is the sealing process. During the sealing, pores of the anodic

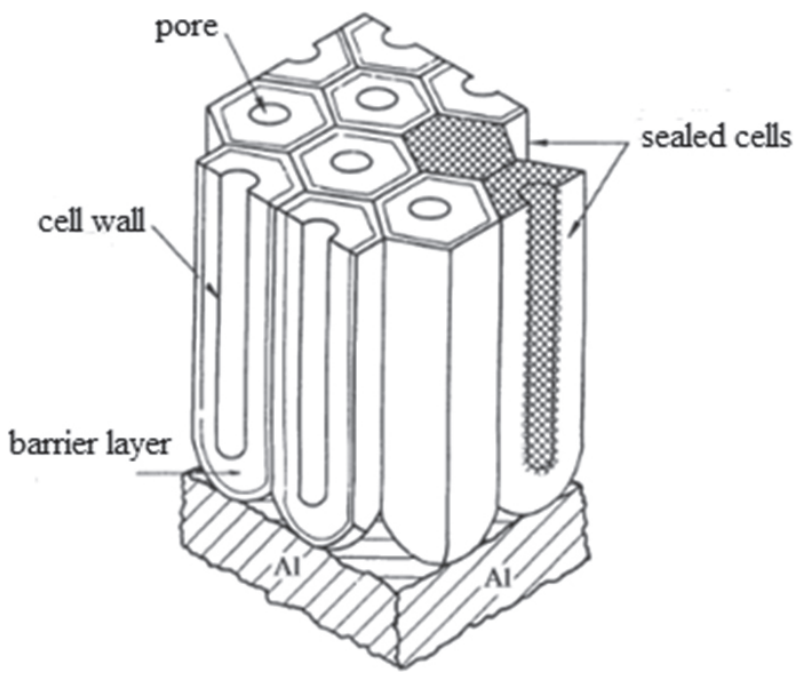

Fig. 1. Structure of aluminium oxide (Brace, Sheasby, 1979).

oxide are closed. This process contributes to anticorrosion protection and enhances the decorativeness of the specimens.

Although the process of electrolytic colouring is routinely used, there is still place for improvement. The scope of this project is the study of parameters controlling the electrolytic colouring of anodized aluminium to form uniform black coatings.

\section{Materials and Methods}

\section{Sample preparation}

An aluminium sheet (Al 99.5) was cut to dimensions of $40 \times 70 \times 1 \mathrm{~mm}$ and subsequently the hinge arm was cut, which served as a conductive connection 
during the pre-treatment operations, anodic oxidation and electrolytic colouring.

\section{Pre-treatment operations}

Surface of these samples was pre-treated in four steps:

1. degreasing was realised in commercial solution Aktigal (Slovakia): in this stage, rough dirt and dust were removed from the surface.

2. alkaline etching, in this step, surface of the samples was subjected to an $\mathrm{NaOH}$ solution of $90 \mathrm{~g} / \mathrm{l}$ and the natural oxide layer was removed.

3. satin etching was done in a solution containing $90 \mathrm{~g} / \mathrm{l}$ of $\mathrm{NaOH}, 109 \mathrm{~g} / \mathrm{l}$ of $\mathrm{NaNO}_{3}$ and $1 \mathrm{~g} / \mathrm{l}$ of sucrose which smoothed the surface.

4. brightening is the last step of the pre-treatment process and it was done in an $\mathrm{HNO}_{3}$ solution of $400 \mathrm{~g} / \mathrm{l}$. Nitric acid removed alloy components from the surface, which produced black spots.

Each step of the pre-treatment process took three minutes at the temperature of $75^{\circ} \mathrm{C}$. After each step, the samples were rinsed with tap water for the time equal to that of the pre-treatment operation and they were left in distilled water before the following treatment. All chemicals used were of the p.a. purity.

\section{Anodic oxidation}

In the process of anodic oxidation, sulphuric acid electrolyte was used. Concentration of sulphuric acid was $200 \mathrm{~g} / 1$ and process current density of $1.5 \mathrm{~A} / \mathrm{dm}^{2}$ was maintained at the temperature of (18-22) ${ }^{\circ} \mathrm{C}$ for the anodization time of 20 minutes. After the anodic oxidation, the samples were rinsed with tap water for the time equal to that of the process and then put into distilled water.

\section{Electrolytic colouring and sealing}

Electrolytic colouring was carried out using alternating current in the commercial solution ALFICOLOR 699. The studied parameters of the process included:

- time of colouring - 9, 12 and 15 minutes;

- AC voltage - in the range of $(12-19 \mathrm{~V})$;

- composition of electrodes - stainless steel AISI

304 marked electrode 1 and AISI 316 marked electrode 2;

- area of electrodes - ratios of the sample area to the counter electrode $2: 1$ and $1: 2$;

- distance between electrodes -2 or $3.5 \mathrm{~cm}$;

- agitation of the electrolyte using a magnetic stirrer.

After the colouring, the samples were rinsed with tap water for 10 minutes, dried by a hot-air dryer ( 2 min both sides of the samples) and subsequently the quality of the coatings was determined by spectrocolorimetry employing parameters $L^{*} a^{*} b^{*}$. This system characterizes any colour by the luminance parameter, $L^{*}$, and two colour coordinates, $a^{*}, b^{*}$, which specify the point on the chromaticity diagram (Fairchild, 2004). Parameter $L^{*}$ represents the colour shade of the finishes and parameters $a^{*}$, $b^{*}$ characterize the brightness of the finishes; for an ideal black body, these parameters are $0 * 0 * 0$. Only the $L^{*}$ parameter is decisive for the coating quality determination and the results are presented in Figs 3-8. A spectrocolorimeter Spectroguide 45/0 (Germany) was used.

Sealing was carried out in distilled water for 10 minutes after the electrolytic colouring.

Thickness of the specimens was measured by a gauge MINITEST $500 \mathrm{~N}$ (Germany) which works on the eddy current principle measuring the insulting coatings on non-ferrous metals. Before the measurement, the gauge was calibrated against an uncoated sample. Presented values of the coating thickness is the mean value of ten readings. The values of layer thickness were measured after anodic oxidation, electrolytic colouring and sealing of the specimens.

\section{Results and discussion}

A study on the parameters for uniform black coatings in the process of tin acidic electrolytic colouring was carried out. Fig. 2 shows the layer thicknesses after anodic oxidation (AO), electrolytic colouring $(\mathrm{AO}+\mathrm{c})$ and sealing $(\mathrm{AO}+\mathrm{c}+\mathrm{s})$. Thickness of the layer after anodic oxidation was found to be about $10.5 \mu \mathrm{m}$. After colouring, the thickness increased, which can be explained by $\mathrm{Al}_{2} \mathrm{O}_{3}$ growth occurring in the anodic half-cycle of the colouring process (Tsangaraki-Kaplanoglou et al., 2006). After the sealing process, thickness of the oxide layer decreased due to pores closing. All the presented results are in good correlation with literature (Wernick et al., 1987).

Quality of the coating was evaluated based on the $L^{*}$ parameter of the spectrocolorimetry coordinates, which was measured after the colouring and sealing processes. The studied parameters of electrolytic colouring are provided in the text below.

First, the influence of the colouring time on the coating quality was analysed in the range of $(8-15) \mathrm{min}$ under constant voltage of $12 \mathrm{~V}$. The colouring time of 9 min (as it is shown in Fig. 3) displays the lowest value of the $L^{*}$ parameter. The value of the $L^{*}$ parameter was 12.0 after the colouring and after the sealing it decreased to 3.9.

The other parameters of the electrolytic colouring were studied at the colouring time of $9 \mathrm{~min}$.

Different composition of the counter electrode was studied as the next parameter. According to the 


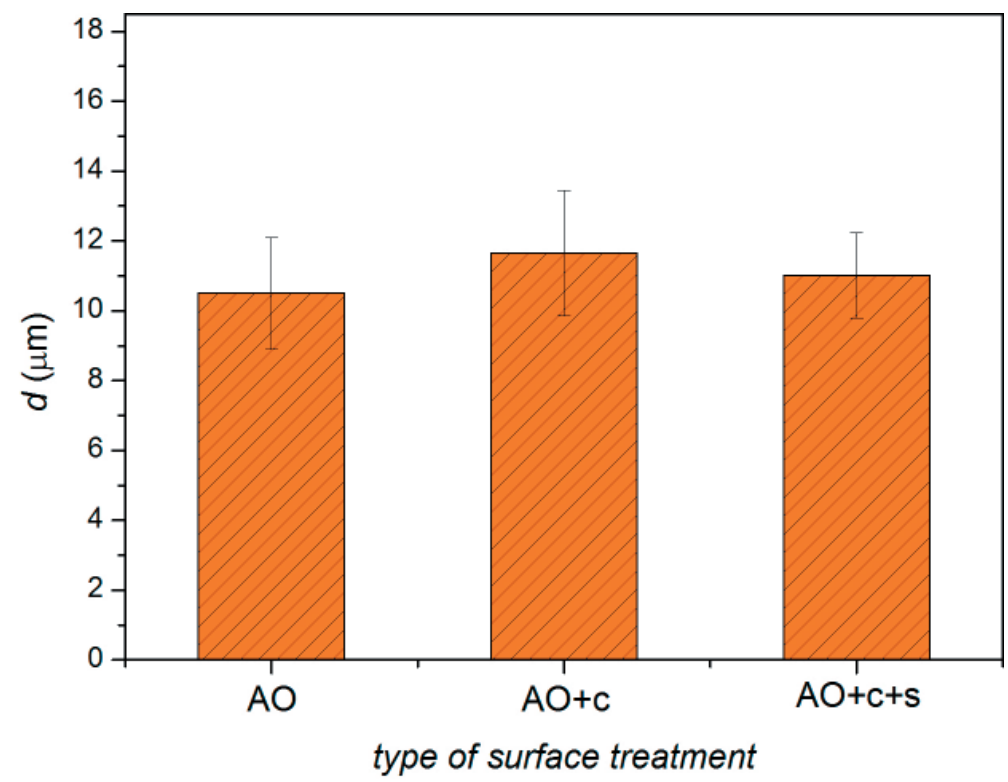

Fig. 2. Average values of thickness measured after anodic oxidation and colouring under the following conditions: electrode 1 , ratio of sample area to electrode area $-1: 2$, distance between sample and electrode $-2 \mathrm{~cm}$, $t=9 \mathrm{~min}$, agitation $=700 \mathrm{rpm}, U=14 \mathrm{~V}$ and sealing time $t=10 \mathrm{~min}$.

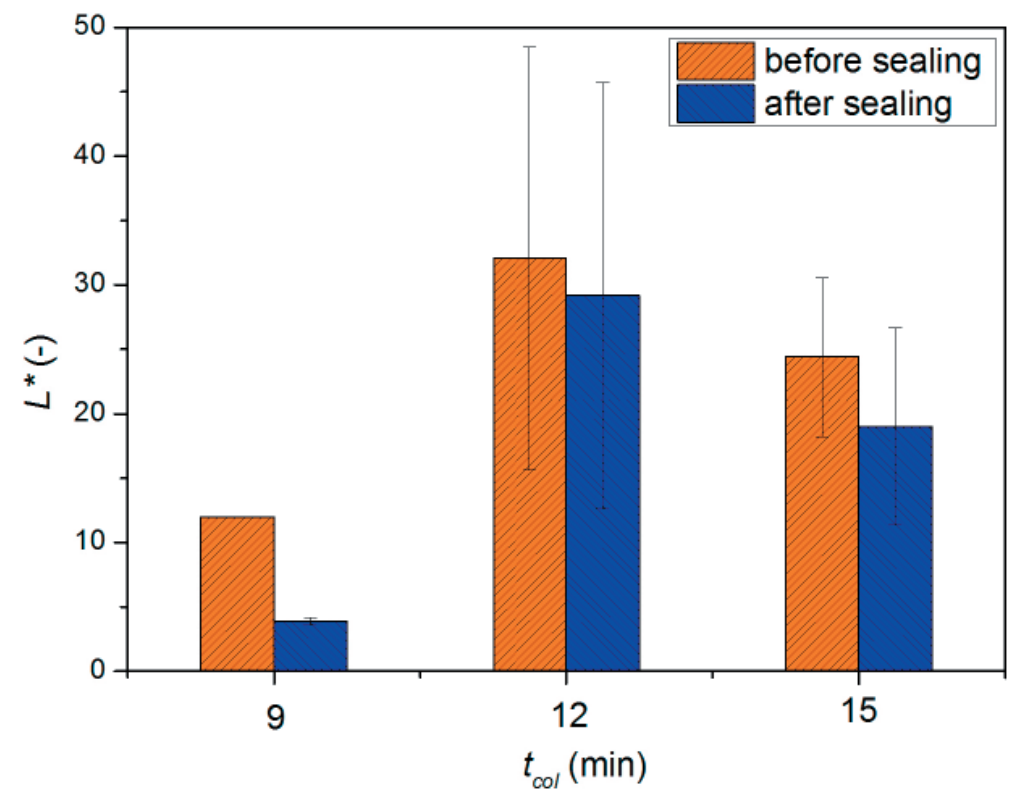

Fig. 3. Dependence of the $L^{*}$ parameter on the colouring time under the following conditions: electrode 1, ratio of sample area to electrode area $-2: 1$, distance between electrodes $-3.5 \mathrm{~cm}, U=12 \mathrm{~V}$, agitation $=700 \mathrm{rpm}$.

results depicted in Figure 4, it can be assumed that the composition of the electrode does not have any effect on the quality of formed coatings. Values of the $L^{*}$ parameter are comparable with those obtained with electrode 1 . It can be also concluded that optimal value of voltage used in this process is $12 \mathrm{~V}$ when the lowest values of the $L^{*}$ parameter were observed.

The following measurements were focused on the different ratio of the sample area to the counter electrode area. The ratio of $1: 2$ was used instead of $2: 1$. Based on the results depicted in Fig. 5 it can be stated that the counter electrode with larger area than that of the sample provides more homogeneous coatings.

Fig. 6. shows the results of $L^{*}$ comparison for the ratio the sample area to the counter electrode area of $2: 1$ and $1: 2$.

It can be clearly seen that the ratio of $1: 2$ of the electrode area results in lower $L^{*}$ values for the coloured finishes. Based on the achieved results, an optimised parameter was applied in the next study - smaller sample area to the counter electrode area. 


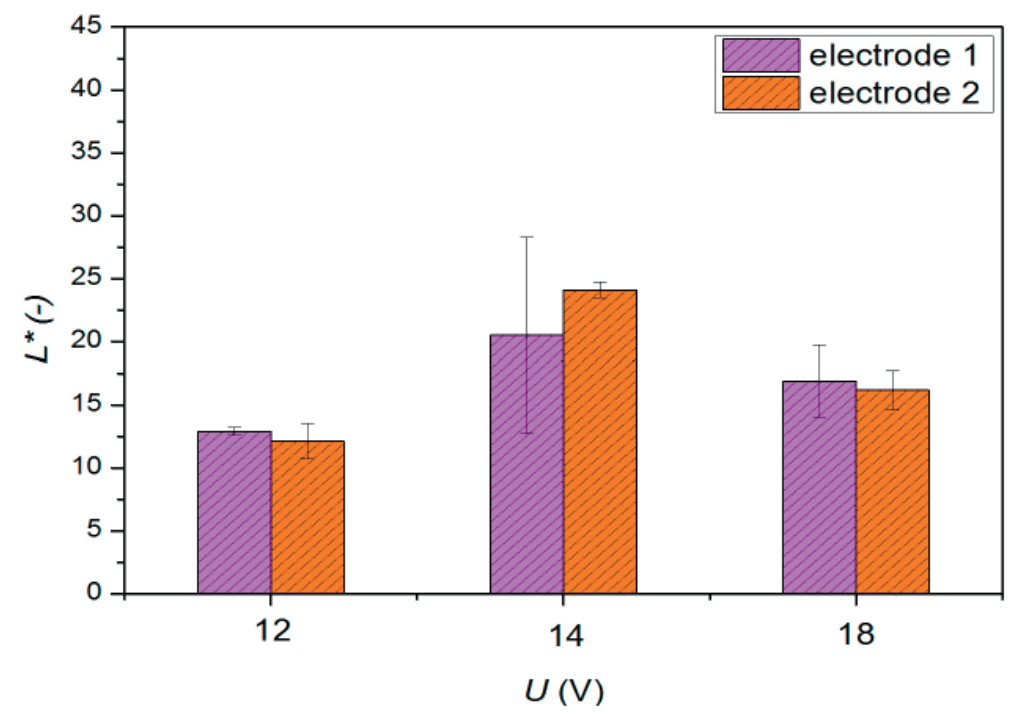

Fig. 4. Comparison of the $L^{*}$ parameter using the counter electrodes with different composition.

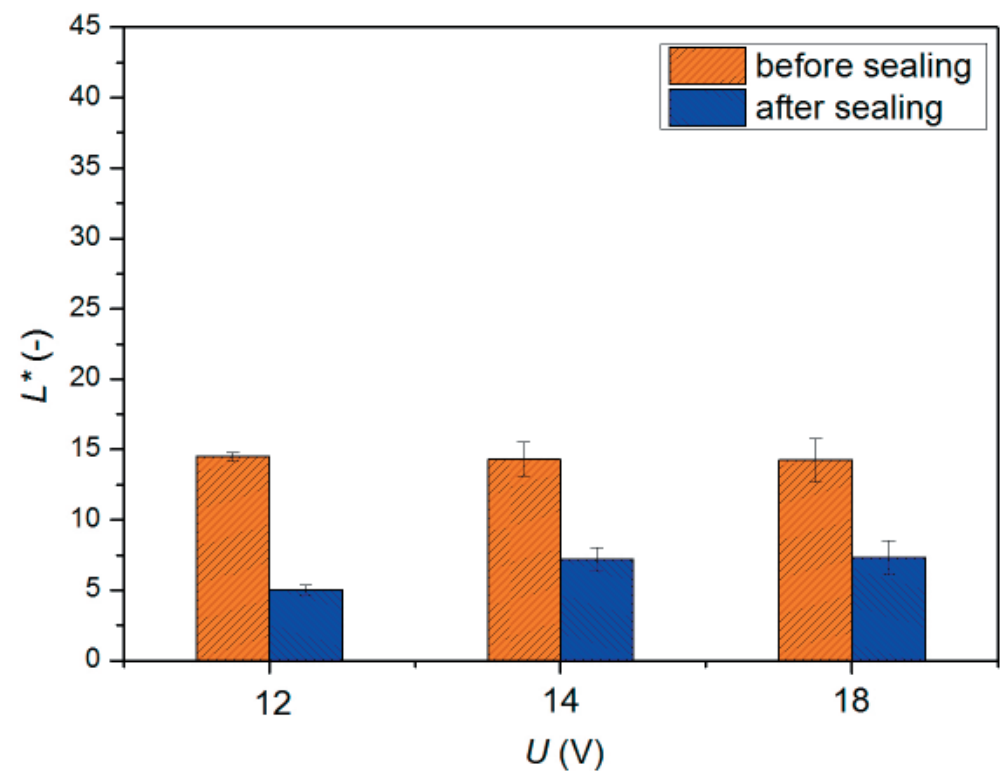

Fig. 5. Dependence of the $L^{*}$ parameter on applied AC voltage under the following conditions: electrode 1 , ratio of sample area to electrode area $-1: 2$, distance between electrodes $-3.5 \mathrm{~cm}$, colouring time $t=9 \mathrm{~min}$, agitation $=700 \mathrm{rpm}$.

Distance between the sample and electrode was another studied parameter. It was reduced from $3.5 \mathrm{~cm}$ to $2 \mathrm{~cm}$, which positively affected the colouring of the anodized aluminium. The values of parameter $L^{*}$ were lower for the electrode distance of $2 \mathrm{~cm}$ in comparison to those at $3.5 \mathrm{~cm}$ as it can be seen in Fig. 7 .

The effect of agitation of the electrolyte on the quality of coatings was studied as well. The realised measurements were carried out at constant rate of agitation of $700 \mathrm{rpm}$ and without agitation. Results for finishes without agitation depicted in Fig. 8 show higher values of the $L^{*}$ parameter compared with the finishes obtained under the same conditions with agitation (Fig. 2-7).
The positive effects of agitation, area of the electrodes and distances between the electrodes are in good accordance with the theory of electrodeposition. All of the studied parameters (agitation, distance between electrodes, and area of the electrodes) contribute to the homogeneity of current distribution during the electrodeposition process (Paunovic and Schlesinger 2006).

On basis of the studied parameters it can be concluded that the electrolytic colouring of anodized aluminium species can be successfully done under the following conditions: AC voltage of $12 \mathrm{~V}$, colouring time of 9 minutes, ratio of sample area to counter electrode area of $1: 2$, distance between electrodes of $2 \mathrm{~cm}$, ensures black coatings of satisfying quality. 


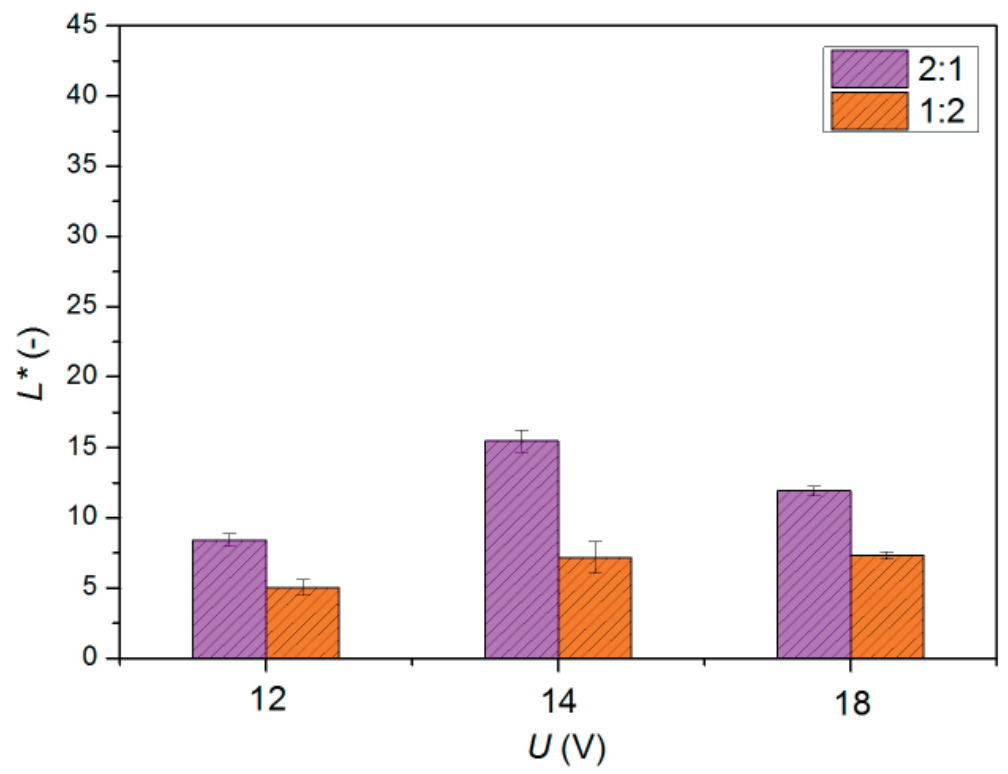

Fig. 6. Comparison of $L^{*}$ parameter at different ratios of sample area to the counter electrode.

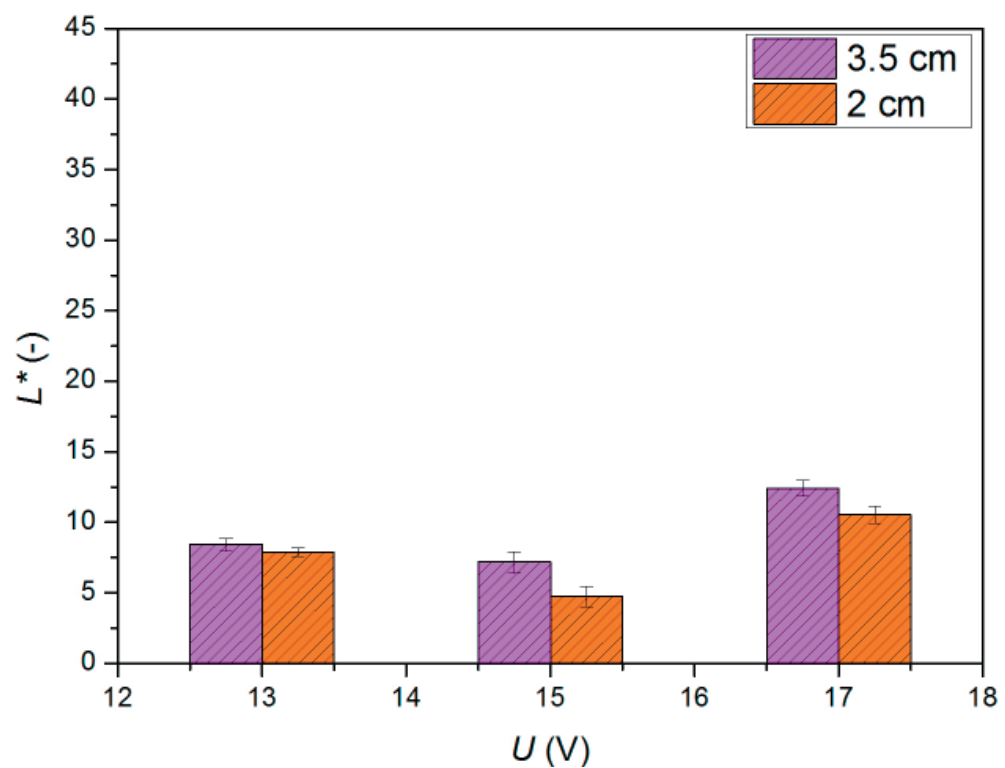

Fig. 7. Comparison of the $L^{*}$ parameter at 3.5 and $2 \mathrm{~cm}$ distance between the sample and the counter electrode.

When applying the optimised parameters of electrolytic colouring for anodized aluminium, the quality of coloured finishes was influenced by the electrolyte due to the instability of tin ions in the acidic electrolyte.

Figure 9 depicts the samples coloured under optimum conditions. It can be seen that the coatings are homogeneous on the whole surface of the samples.

\section{Conclusions}

The objective of the study was to find optimum conditions for the electrolytic colouring of anodized aluminium utilising electrolytes containing tin ions. Parameters influencing the quality of the electrolytic colouring process were studied to reach uniform black coatings.

It was found that $\mathrm{AC}$ voltage of $12 \mathrm{~V}$, colouring time of 9 minutes, ratio of sample area to counter electrode area of $1: 2$, and distance between electrodes of $2 \mathrm{~cm}$ ensure black coatings with satisfying quality. The most important parameter under the studied conditions is the electrolyte freshness due to the instability of tin ions in the acidic electrolyte in time.

Quality of the coloured finishes was improved by the sealing process which contributes to the increase in the corrosion protection as well as the decorativeness of the material. 


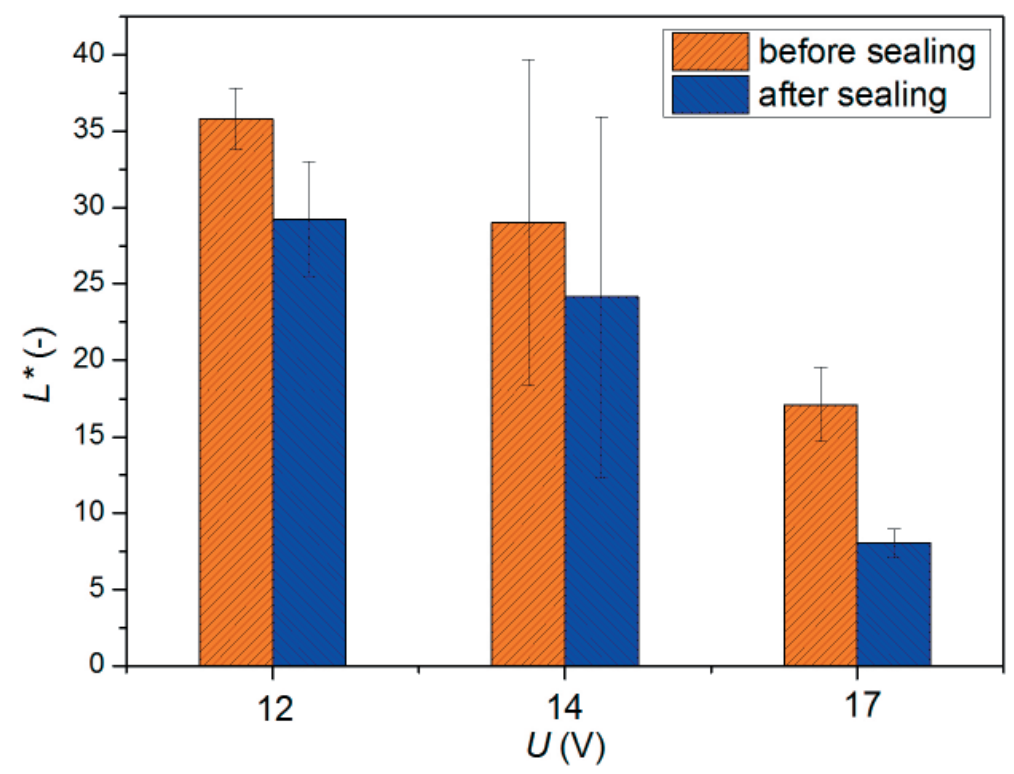

Fig. 8. Dependence of the $L^{*}$ parameter on applied AC voltage under the following conditions: electrode 1 , ratio of sample area to electrode area $-1: 2$, distance between electrodes $-2 \mathrm{~cm}$, colouring time $t=9 \mathrm{~min}$, without agitation.
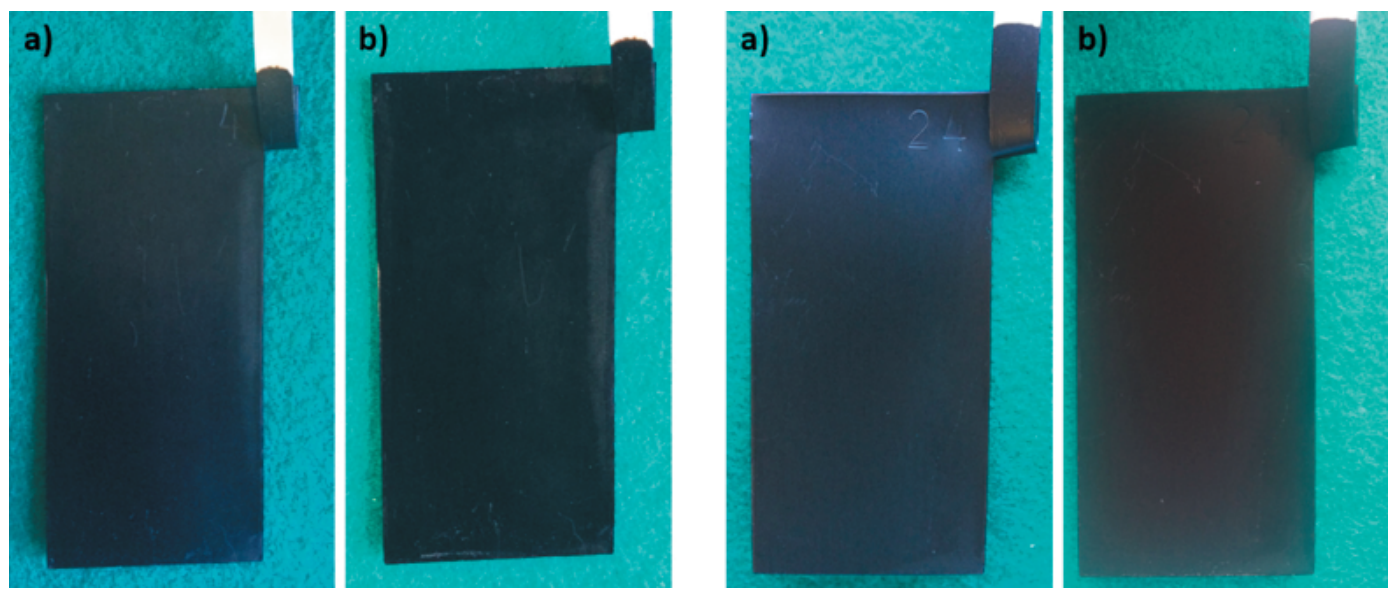

Fig. 9. Samples coloured under optimal conditions: a) before sealing, b) after sealing.

\section{Acknowledgement}

Financial support by the Ministry of Education, Science, Research and Sport of the Slovak Republic within the project VEGA 1/0792/17 is greatly acknowledged.

\section{References}

Brace AW, Sheasby PG (1979) The Technology of Anodizing Aluminium. Technicopy Ltd, England.

Fairchild MD (2005) Color and Image Appearance Models, Chichester UK, John Wiley and Sons.

Cheng CH (2015) Electro- Chemo- Mechanics of Anodic Porous Alumina Nano- Honeycombs: Self- ordered Growth and Actuation. The University of Hong Kong, Hong Kong.

Michna S̆, Lukáč I et al. (2005) Encyclopedia of Aluminium. Adin, s.r.o, Prešov.
Paunovic M, Schlesinger M (2006) Fundamentals of Electrochemical Deposition. John Wiley \& Sons, Inc. (USA) Hoboken, New Jersey.

Srinivasan B (2005) Troubleshooting of Electrolytic Color Anodizing of Aluminium. USA.

Švorc J (2016) Sealing of Anodized Aluminium and its Alloys. České vysoké učení technické v Praze, Prague.

Tsangaraki-Kaplanoglou I. et al. (2006) An Investigation of Electrolytic Coloring Process of Anodized Aluminium Coatings. Surface \& Coatings Technology: 2749-2759.

Wernick S, Pinner R, Sheasby PG (1987) The Surface treatment and Finishing Aluminium and its Alloys. Vol. 1 and 2. ASM International Finishing Publications Ltd, England. 